\title{
The STAT3 inhibitor S3I-201 suppresses fibrogenesis and angiogenesis in liver fibrosis
}

\author{
Zhuo Wang ${ }^{1,2} \cdot$ Jia'an $\mathrm{Li}^{2} \cdot$ Wen'ang Xiao ${ }^{2} \cdot$ Jiafu Long $\mathbb{1}^{1,3} \cdot$ Hongmin Zhang $\mathbb{D}^{2}$
}

Received: 12 May 2018 / Revised: 3 August 2018 / Accepted: 3 August 2018 / Published online: 11 September 2018

(c) United States \& Canadian Academy of Pathology 2018

\begin{abstract}
Liver fibrosis is a common pathological response to chronic hepatic injury. STAT3 is actively involved in the fibrogenesis and angiogenesis seen in liver fibrosis. S3I-201 (NSC 74859) is a chemical inhibitor of STAT3 activity, which blocks the dimerization of STAT3, STAT3-DNA binding and transcription activity. This study evaluated the effects of S3I-201 against liver fibrosis. S3I-201 inhibited the proliferation, migration, and actin filament formation in primary human hepatic stellate cells (HSCs), as well as the expression of $\alpha$-SMA, collagen I and TIMP1 in both primary HSC and in a $\mathrm{CCl}_{4}$-induced fibrosis mouse model. S3I-201 induced both apoptosis and cell cycle arrest in the HSC cell line (LX-2). S3I-201 inhibited the expression of fibrogenesis factors TGF $\beta 1$ and TGF $\beta$ RII, as well as the downstream phosphorylation of Smad2, Smad3, Akt and ERK induced by TGF $\beta 1$. In addition to fibrogenesis, both in vitro and in vivo assays showed that S3I-201 inhibited angiogenesis through expression suppression of VEGF and VEGFR2. Moreover, S3I-201 also had a synergistic effect with sorafenib, an FDA approved liver cancer drug, in the proliferation, apoptosis, angiogenesis and fibrogenesis of HSC. S3I-201 suppressed liver fibrosis through multiple mechanisms, and combined with sorafenib, S3I-201 could be a potentially effective antifibrotic agent.
\end{abstract}

\section{Introduction}

Liver fibrosis is a common pathological consequence of chronic liver disease [1], and activated HSCs play a pivotal role in the development of liver fibrosis. HSCs are activated

Electronic supplementary material The online version of this article (https://doi.org/10.1038/s41374-018-0127-3) contains supplementary material, which is available to authorized users.

Zhuo Wang

wangz3@sustc.edu.cn

$\triangle$ Hongmin Zhang

zhanghm@sustc.edu.cn

hongmin_zhang@foxmail.com

1 College of Life Sciences, Nankai University, Tianjin 300071, China

2 Department of Biology, Guangdong Provincial Key Laboratory of Cell Microenvironment and Disease Research, Shenzhen Key Laboratory of Cell Microenvironment and SUSTech-HKU Joint Laboratories for Matrix Biology and Diseases, Southern University of Science and Technology, Shenzhen 518055, China

3 State Key Laboratory of Medicinal Chemical Biology, Nankai University, Tianjin 300071, China after chronic liver injury, and develop a myofibroblast ( $\alpha$-SMA)-like phenotype that proliferate, become fibrogenic and produce increased amount of extracellular matrix proteins [2]. Hepatic fibrosis is characterized by dysregulated changes of extracellular matrix (ECM), including the net increased synthesis of collagens, especially type I, and the decreased matrix degradation. Degradation of ECM is associated with ECM-removing matrix metalloproteinases (MMPs), which is inhibited partially by enhanced expression of tissue inhibitors of metalloproteinases (TIMPs) in liver fibrosis [3]. To date, no approved antifibrotic therapy is in clinical use, largely owing to the various side effects and uncertain positive clinical outcomes [4, 5]. Liver cirrhosis, as the end-stage of liver fibrosis, has a high mortality with liver transplantation as the only curative therapy. Therefore, effective anti-fibrotic therapeutics are required urgently.

Several cytokines play critical roles in progression of HSC activation and fibrosis, particularly transforming growth factor $\beta 1$ (TGF $\beta 1$ ) [6]. TGF $\beta 1$ drives the transdifferentiation of phenotypical HSCs from quiescence to activation through paracrine and autocrine mechanisms [7]. TGF $\beta 1$ also induces collagen I expression and $\alpha$-SMA stress fiber organization directly [8]. Recent study shows that TGF- $\beta 1$ is the pivotal profibrogenic cytokine [9] and strategies targeting at 
disrupting TGF $\beta 1$ action markedly attenuated liver fibrosis in experimental models [10]. Therefore, inhibiting signaling transduction by TGF $\beta 1$ might be an effective therapy for hepatic fibrosis. TGF $\beta 1$ is a target gene of signal transducer and activator of transcription 3 (STAT3) [11] that promotes hepatic fibrosis through increasing TGF- $\beta 1$ expression in liver fibrotic mouse model [12]. The rapid early activation of STAT3 has been observed in HSCs and fibroblasts but not in normal hepatocytes [9], indicating that STAT3 might be a therapeutic target for liver fibrosis.

Angiogenesis, the sprout of new vessels from preexisting ones, increases significantly in many chronic liver diseases including liver fibrosis [13]. Both angiogenesis and fibrogenesis are crucial components in the evaluation of liver disease progression, and the association between them is important for search of therapeutic targets. HSCs promote angiogenesis in liver fibrosis through upregulating the expression of pro-angiogenic factors such as vascular endothelial growth factor (VEGF) and VEGF receptors (VEGFR) [14]. And inhibition of neovascularization by disrupting VEGF signaling may attenuate the progression of liver fibrosis in $\mathrm{CCl}_{4}$-induced mouse model [14]. VEGF is well known as a STAT3 target, indicating the key role of STAT3 in promoting angiogenesis [15]. Therefore, inhibiting STAT3 may attenuate angiogenesis and furthermore fibrogenesis, one more support of STAT3 as a potential therapeutic target for liver fibrosis.

S3I-201 (NSC 74859) is an inhibitor of STAT3 activity [16], which blocks the phosphorylation and dimerization of STAT3 and thereafter STAT3-activated transcription. As STAT3 plays important roles in both angiogenesis and fibrogenesis, blocking the activation of STAT3 might be an attractive therapeutic strategy in liver fibrosis treatment. In this study, we explored whether S3I-201 exerts any effect on the activation of HSCs in response to TGF $\beta 1$ and VEGF, and also the effects on anti-fibrogenesis and antiangiogenesis in experimental fibrosis models.

\section{Materials and methods}

\section{Drugs}

S3-201 and Sorafenib (Selleck chemicals, USA) were dissolved in DMSO. For in vitro assays, the concentration of DMSO was below $0.01 \%(\mathrm{v} / \mathrm{v})$. For in vivo studies, S3I-201 was further diluted with saline solution and the concentration of DMSO was below $0.001 \%$.

\section{Cell culture}

Primary human HSCs were obtained from Science Research Laboratories (Carlsbad, CA, USA) and cultured in DMEM with $10 \%$ FBS. Experimental manipulations were performed with cells at passage 4-5. Immortalized human HSC cell line (LX-2) was cultured according to previous report [17].

\section{Experimental fibrosis model by $\mathrm{CCl}_{4}$ administration}

Male Balb/c mice, aged 6 weeks, were purchased from Charles River Laboratories (Beijing, China). The research protocol was approved by the Institutional Animal Care and Use Committee at the Southern University of Science and Technology. All fibrotic experimental groups received $2 \mathrm{ml} / \mathrm{kg}$ of $\mathrm{CCl}_{4}$ dissolved in $150 \mu \mathrm{l}$ olive oil twice a week. Liver fibrosis was induced by $\mathrm{CCl}_{4}$ administration for 2 weeks, whereas mice that received olive oil injection only for 2 weeks served as sham controls. The treatment group was administered $\mathrm{CCl}_{4}$ for 2 weeks and then administered S3I-201 $5 \mathrm{mg} / \mathrm{kg}$ every other day, together with $\mathrm{CCl}_{4}$ injection for another 2 weeks. Mice injected with $\mathrm{CCl}_{4}$ for 4 weeks were included for monitoring the development of liver fibrosis. Liver tissues and blood were collected for subsequent experiments.

\section{Histological and immunohistochemical studies}

Liver samples were formalin-fixed, paraffin-embedded, sectioned at $4 \mu \mathrm{m}$, and processed routinely for Sirius-red staining [17]. Immunohistochemical staining of $\alpha$-SMA was performed as previously described [18]. The area of positive staining was quantified using a computer-aided image analysis system NIS Elements Advanced Research (Nikon, Tokyo, Japan). For detection of angiogenesis in vivo, liver samples were immunostained with anti-CD31 monoclonal antibody (MXB Biotechnologies, China) according to previous description [18].

\section{Serum chemisty}

Total bilirubin (TBil), aspartate aminotransferase (AST), alanine aminotransferase (ALT), and serum albumin (ALB) were measured using standard laboratory assays.

\section{Wound-healing assay}

For measurement of cell migration during wound healing, primary human HSCs were seeded in 6-well plates and grown to confluence in a growth medium containing $10 \%$ FBS. Confluent HSC were deprived of serum for $24 \mathrm{~h}$, and then disrupted to generate a linear wound, followed by incubation in a medium with or without S3I-201 for $24 \mathrm{~h}$. HSC were subsequently fixed and observed under phase contrast microscopy. Experiments were carried out in duplicate, and five fields of well were recorded. 


\section{Cell proliferation assay}

Cells were seeded into 96-well plate and cultured overnight, treated with drugs at the indicated concentrations. Cell proliferation was measured at $48 \mathrm{~h}$ after treatment using 3-(4, 5-dimethylthiazol-2-yl)-5-(3-carboxymethoxyohenyl)-2-(4-sulfophenyl-2H-tetrazolium) (MTS) assay (Promega Co., Madison, WI). The result was analyzed by Graphpad Prism 5.

The Chou-Talalay median effect analysis [19] was used to evaluate the potential synergistic growth inhibitory effects of S3I-201 and sorafenib. LX-2 cells were treated for $48 \mathrm{~h}$ with combinations of S3I-201 and sorafenib at indicated ratio. MTS assay was used to measure the cell proliferation. The combination index (CI) was calculated using the CompuSyn software (ComboSyn, Inc.). The CI values of $<1,=1$, and $>1$ indicated synergism, additive effects, and antagonistic effects, respectively.

\section{Effect of S3I-201 on H-HSC actin cytoskeleton}

H-HSC were treated with incremental concentrations of S3I-201 for $24 \mathrm{~h}$. Cells were then fixed with $4 \%$ paraformaldehyde for $5 \mathrm{~min}$ at room temperature, and then permeabilized with $0.1 \%$ triton $\mathrm{X}-100$ for another $5 \mathrm{~min}$. After washing, cells were stained with $50 \mu \mathrm{g} / \mathrm{ml}$ fluorescent phalloidin conjugate (Sigma, St Louis, MO, USA) at room temperature for $40 \mathrm{~min}$, then washed 4-5 times with PBS, and covered with ProLong Gold Antifade Reagent with DAPI (Cell Signaling Technology, Danvers, MA, USA). Actin cytoskeleton was observed using Leica TCS SP8 confocal microsystems (Wetzlar, Germany).

\section{Detection of apoptosis by flow cytometry}

LX-2 cells seeded in 6-well plates were treated with different concentrations of S3I-201 for $24 \mathrm{~h}$. Cells were then harvested and stained with Annexin V-FITC Apoptosis Detection Kit (BD Pharmingen, San Diego, CA) according to the manufacture's protocol. Cells were analyzed by flow cytometry.

\section{Detection of cell cycle by flow cytometry}

LX-2 cells were plated out into 6-well plate and exposed to incremental concentrations of S3I-201 for $24 \mathrm{~h}$. Cells were fixed with $70 \%$ iced ethanol, followed by PI/RNase Staining Buffer Solution (BD Pharmingen, San Diego, CA). Cells were analyzed by flow cytometry (Beckman). The percentage of cell phases were assessed by ModFit LT software (Verity Software House, Topsham, ME).
Effect of S3I-201 on VEGF secretion in LX-2 cells

LX-2 cells were seeded into 6 -well plates and treated with different concentrations of S3I-201 for $24 \mathrm{~h}$. Cell culture supernatants were collected and centrifuged, and the protein levels of VEGF were measured by Human VEGF Quantikine ELISA Kit (R\&D System, Minneapolis, MN) according to manufacturer's protocol. For analysis of VEGF induced by TGF $\beta 1$, LX- 2 cells were starved overnight and then stimulated with different concentration of TGF $\beta 1$ for $6 \mathrm{~h}$, and cell culture supernatants were collected and analyzed by Human VEGF Quantikine ELISA Kit.

\section{Confocal immunofluorescence microscopy}

Cells were seeded into confocal dish, then treated with S3I-201 for $24 \mathrm{~h}$. Cells were fixed with $4 \%$ paraformaldehyde for $10 \mathrm{~min}$, washed three times in PBS, permeabilized with iced-methanol for $10 \mathrm{~min}$ at $-20^{\circ} \mathrm{C}$, and further washed three times with PBS. Cells were then blocked in $1 \%$ bovine serum albumin (BSA) for $30 \mathrm{~min}$ and incubated with phosphor-STAT3 or/and $\alpha$-SMA antibody at $4{ }^{\circ} \mathrm{C}$ overnight. Subsequently, cells were rinsed three times in PBS, incubated with Alexa fluor 488 rabbit and Alexa fluor 594 mouse for $1 \mathrm{~h}$ at room temperature in the dark, then washed three times with PBS, and covered with ProLong Gold Antifade Reagent with DAPI (Cell Signaling Technology, Danvers, MA, USA). Expression of $\alpha$-SMA and phosphor-STAT3 was observed using Leica TCS SP8 confocal microsystems (Wetzlar, Germany).

\section{Western blots}

Whole cell lysates were prepared using lysis buffer (Cell signaling Technology, Danvers, MA, USA) and the expression levels of $\alpha$-SMA, collagen I, TIMP1, cyclin A, cyclin D1, p21, Bcl-2, Bcl- $\mathrm{x}_{\mathrm{L}}$, cleaved caspase 3, VEGF, VEGFR1, VEGFR2, TGF $\beta$ RI, TGF $\beta$ RII, phosphor-STAT3, phosphor-Smad2, phosphor-Smad3, phosphor-Akt, and phospho-ERK were examined. For liver samples, tissues were homogenized and lysed using lysis buffer. Equal amounts of solubilized proteins were separated by SDS-PAGE and transferred to membrane. After blocking, membranes were immunoblotted with antibodies overnight at $4{ }^{\circ} \mathrm{C}$, followed by detection using HRP-conjugated secondary antibodies (Santa Cruz Biotechnology) at room temperature for $1 \mathrm{~h}$. The immunoreactive proteins were visualized by Western ECL Blotting Substrates (Bio-Rad, Hercules, California). 


\section{Statistical analysis}

Data shown were triplicate of experiments and expressed as mean \pm SEM. One-way ANOVA or two-tailed student's $t$-test was used where appropriate. A $p$-value $<0.05$ was considered statistically significant.

\section{Results}

\section{S3I-201 reduced the level of p-STAT3 and the translocation of p-STAT3 in LX-2 cells}

We first examined the expression of phosphor-STAT3 (p-STAT3) in primary human HSC and mouse liver specimens. Double staining of $\alpha$-SMA and p-STAT3 showed high expression of p-STAT3 in primary human HSC (Fig. 1a). IHC staining of liver specimens from the fibrotic mouse model revealed higher p-STAT3 expression in HSC in fibrotic areas than that in hepatocytes in adjacent nonfibrotic areas (Fig. 1b). S3I-201 blocks the phosphorylation and dimerization of STAT3 and thereafter the translocation from cytosol to nucleus. To confirm the inhibitory effect of S3I-201 on STAT3, we determined the phosphorylation level of STAT3 and translocation of p-STAT3 using human fibrotic cell line LX-2. As shown in Fig. 1, S3I-201 treatment greatly decreased the formation of p-STAT3 (Fig. 1c) and blocked the translocation (Fig. 1d).

\section{S3I-201 inhibited the activation of primary human HSC in culture}

To evaluate the effect of S3I-201 on human HSC (H-HSC) activation, H-HSC were incubated with S3I-201 for $48 \mathrm{~h}$ and typical activation characterization was performed and recorded. As shown in Fig. 2, the proliferation of H-HSC was inhibited by S3I-201 in a dose-dependent manner (Fig. 2a) and the synthesis of $\alpha$-SMA, collagen I and TIMP-1 were all significantly reduced after S3I-201 treatment (Fig. 2b). S3I201 also altered the cell shape and disrupted the wellorganized F-actin bundles in activated HSC (Fig. 2c), as well as inhibited the migration activity of H-HSC (Fig. 2d).

\section{S3I-201 attenuated liver fibrosis in vivo}

To further evaluate the antifibrotic efficacy of S3I-201 in vivo, the $\mathrm{CCl}_{4}$-induced fibrosis mouse model was treated with S3I-201. Fibrosis was observed in all experimental mice after 2 weeks of $\mathrm{CCl}_{4}$ injections and became severe after 4 weeks (Fig. 3a). S3I-201 treatment reduced the extent of liver fibrosis significantly (Fig. 3a), as shown by Sirius red staining of collagen I (Fig. 3b). The antifibrotic effect of S3I-201 in $\mathrm{CCl}_{4}$-treated mice was further confirmed at the molecular level where the expression levels of in situ $\alpha$-SMA (Fig. 3c), collagen I and TIMP-1(Fig. 3d) were remarkably suppressed in the S3I-201-treated group compared to the untreated group. The levels of serum fibrosis markers, TBil, AST, ALT, and ALB, were halted or even reduced after S3I-201 treatment (Fig. 3e), indicating a clear anti-inflammatory activity, a beneficial effect for the treatment of hepatic injury and fibrosis.

\section{S3I-201 induced S phase arrest and apoptosis in LX-2 cells}

To understand why S3I-201inhibits the proliferation of HSCs (Fig. 2a), we examined the effect of S3I-201 on the cell cycle by flow cytometry analysis using LX-2 cells. S3I201 treatment significantly increased the number of cells in $S$ phase (Fig. 4a, b). The molecular basis of $S$ phase arrest induced by S3I-201 was revealed by the increased expression of cyclin dependent kinase inhibitor p21 and decreased expression of cyclin D1 and cyclin A, which regulates the S phase to G2 phase transition (Fig. 4c).

In addition to inducing cell cycle arrest, S3I-201 also significantly increased the level of apoptosis in LX-2 cells as evaluated by Annexin V-FITC and PI staining (Fig. 4d, e). To evaluate the pro-apoptotic signaling pathway activated following S3I-201 incubation, lysates of LX-2 cells after treatment by S3I-201 for $24 \mathrm{~h}$ were analyzed by Western blot. As shown in Fig. 4f, S3I-201 pretreatment reduced $\mathrm{Bcl}-\mathrm{xL}$ and $\mathrm{Bcl}-2$ protein levels and increased the cleavage of caspase 3 .

\section{S3I-201 downregulated the endogenous expression of TGF $\beta 1$ and TGF $\beta R I I$ in LX-2 cells}

TGF $\beta 1$ is a pivotal cytokine in profibrogenesis. The effects of S3I-201 on TGF $\beta 1$ expression and TGF $\beta 1$ regulated signaling were also assessed. As shown in Fig. 5a, the secretion of TGF $\beta 1$ by LX-2 was downregulated by S3I-201 in a dose-dependent manner. Additionally, the expression of TGF $\beta$ RII but not TGF $\beta$ RI was also downregulated upon S3I-201 treatment (Fig. 5b). TGF $\beta 1$ significantly upregulated the phosphorylation of Smad2 and Smad3, and to a less extent, ERK and Akt in LX-2 cells, and S3I-201 treatment reversed the upregulation stimulated by TGF $\beta 1$ (Fig. 5c).

\section{S3I-201 inhibited angiogenesis both in vitro and in vivo}

The effect of S3I-201 on angiogenesis was evaluated in both acell line and the $\mathrm{CCl}_{4}$-induced fibrotic mouse model. As shown in Fig. 6a, the secretion of VEGF, the most potent angiogenesis modulating factor, was suppressed in a dosedependent manner upon S3I-201 treatment on LX-2 cells. The 
A

human HSC

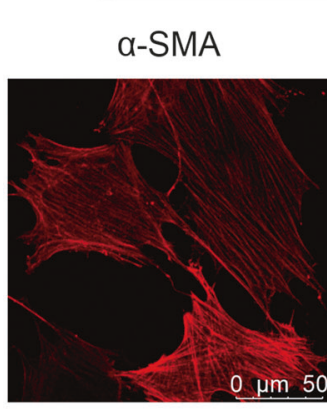

$\mathrm{B}$

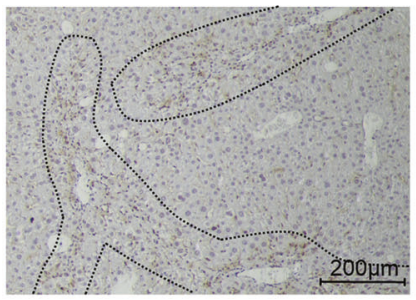

C

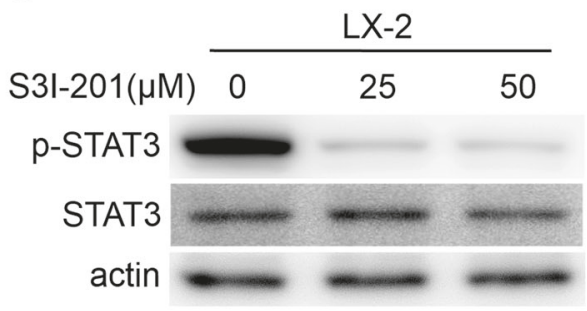

Merge
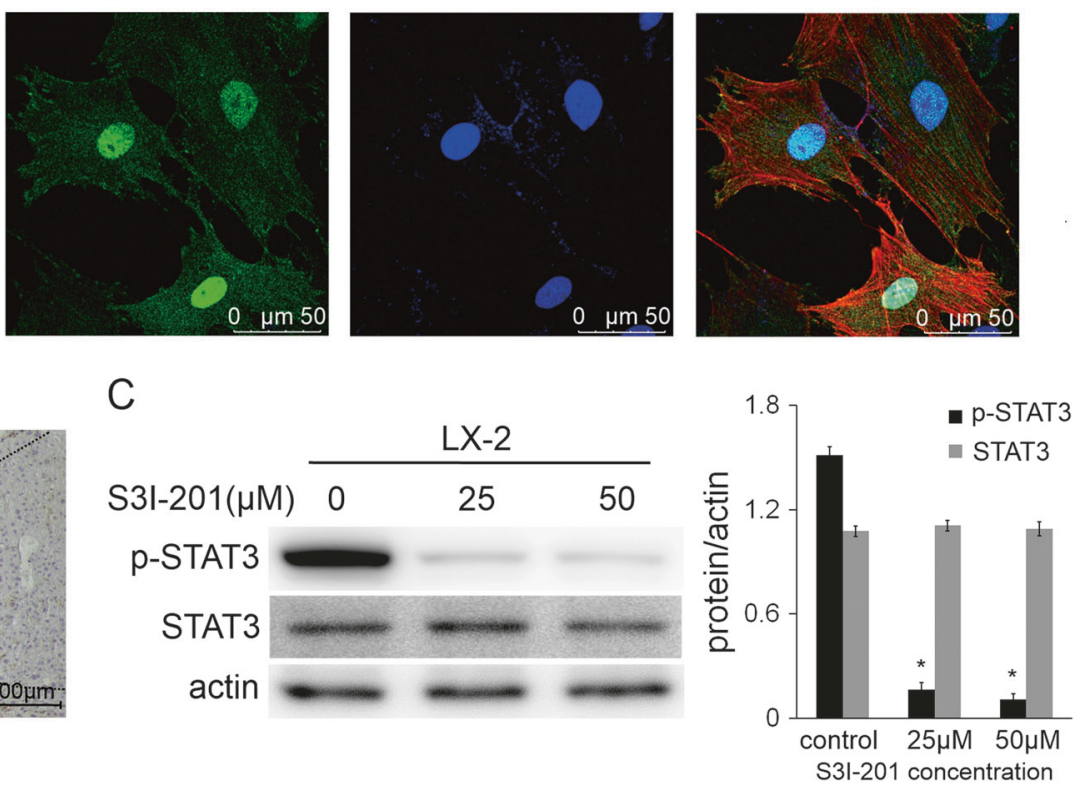

D

LX-2
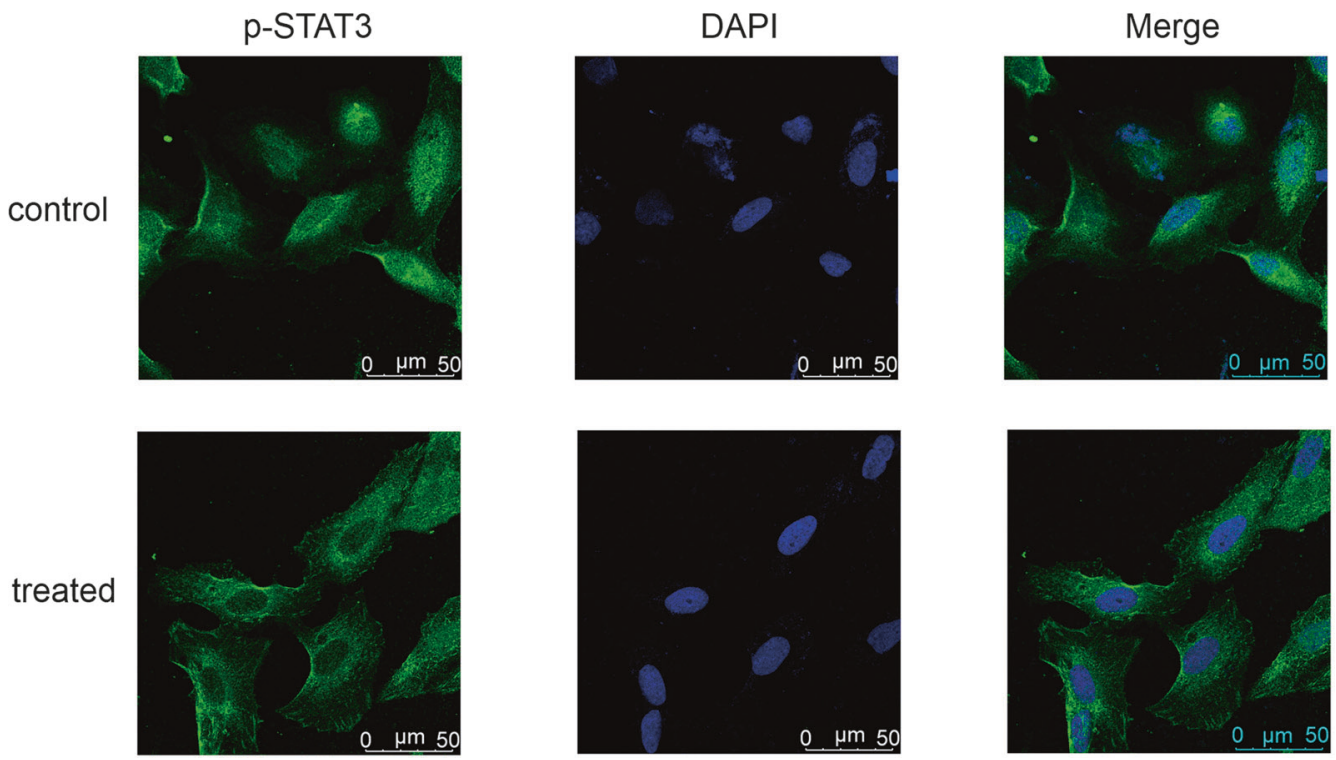

Fig. 1 S3I-201 inhibited the activation and translocation of STAT3 in LX- 2 cells. a $\alpha$-SMA and p-STAT3 were stained by double immunofluorescence staining in primary human HSC. b IHC was applied to check p-STAT3 on mouse fibrotic liver specimen. c Western blot analysis was used to examine the expression of p-STAT3 in LX-2

cells. Cells were treated with S3I-201 for $24 \mathrm{~h}$ and whole lysate was analyzed for the protein expression. $\mathbf{d}$ Translocation of p-STAT3 upon S3I-201 treatment. Cells were treated with the inhibitor for $24 \mathrm{~h}$ and the location of p-STAT3 was stained and determined by confocal

protein levels of VEGFR1 and VEGFR2 were also downregulated by S3I-201 treatment (Fig. 6b). VEGF induced the phosphorylation of Akt and p38 while S3I-201 treatment inhibited this upregulation (Fig. 6c). To further evaluate the effect of S3I-201 in neo-vascularization, the expression level of CD31, an endothelial marker of angiogenesis, was stained and measured in fibrotic septa (Fig. 6d). CD31 levels increased with $\mathrm{CCl}_{4}$ treatment, whereas S3I-201 significantly decreased the percentage of CD31-positive cells. Similar to the LX-2 cell line, the expression levels of VEGFR1 and VEGFR2 in fibrotic tissue of $\mathrm{CCl}_{4}$-induced mouse model were also downregulated (Fig. 6e). 
A Effect of S3I-201 on primary
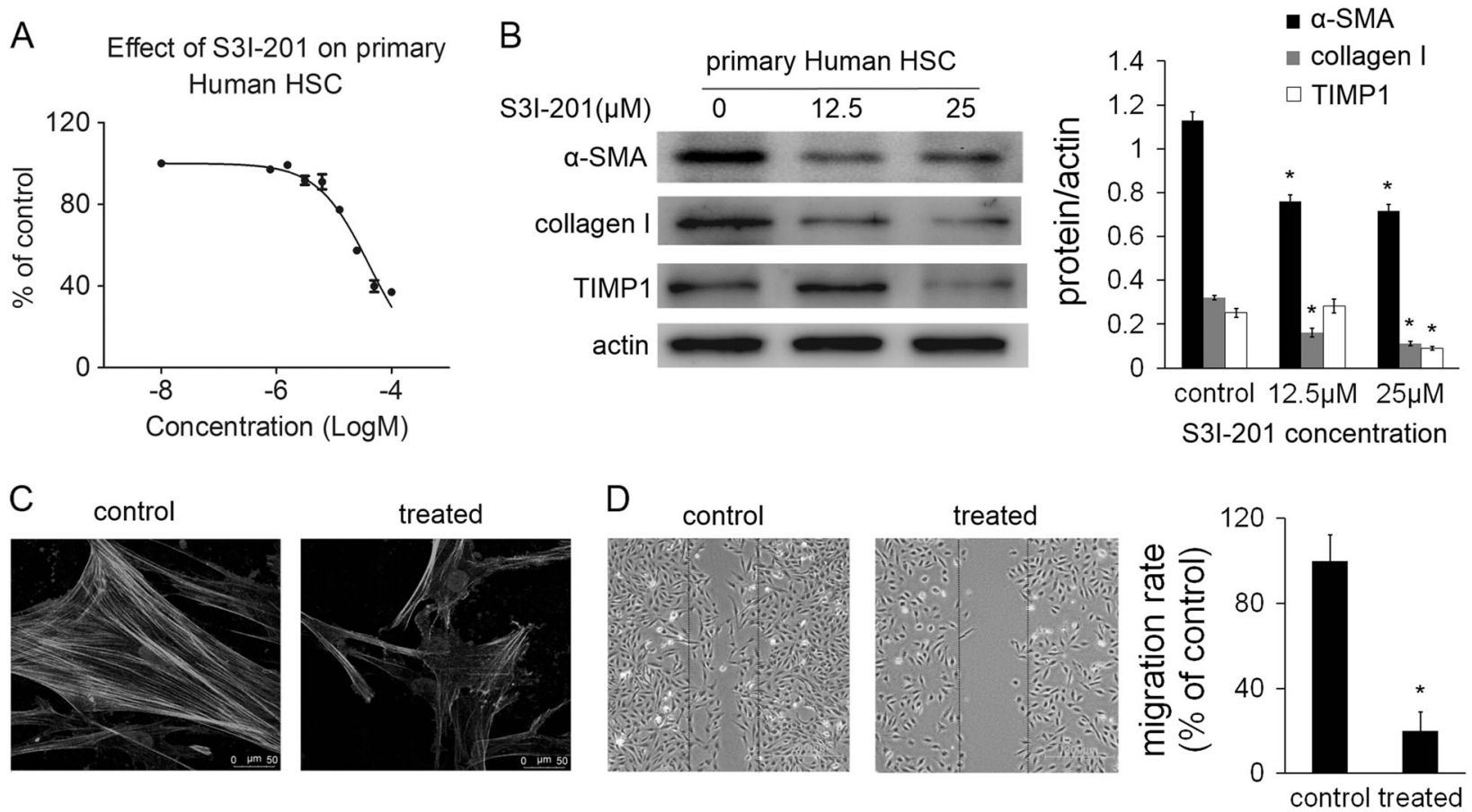

Fig. 2 S3I-201 inhibited the activation of primary human HSC. a Proliferation inhibition of H-HSC by S3I-201. H-HSC was cultured in the presence of different doses of S3I-201 for $48 \mathrm{~h}$, and the proliferation rate was measured by MTS assay. Data are expressed as mean \pm SEM. b S3I-201 downregulated the expression of profibrotic ECM factors. H-HSC was treated with different concentrations of S3I-201 for $24 \mathrm{~h}$. Western blot analysis revealed that the expression of $\alpha$-SMA, collagen I and TIMP1 was significantly reduced by S3I-201. c S3I-201 inhibited cytoskeleton re-arrangement in H-HSC.

\section{S3I-201 reduced VEGF expression induced by exogenous TGF $\beta 1$}

Because of the important roles of TGF $\beta 1$ in profibrogenesis and VEGF in angiogenesis, we also tested the effect of exogenous TGF $\beta 1$ on the expression of VEGF in LX-2 cells. We noticed that not only the total expression of VEGF but also the secretion of VEGF increased in a dosedependent manner upon TGF $\beta 1$ treatment (Fig. 7a), and the increases for both total and secreted VEGF were downregulated by S3I-201 treatment (Fig. 7b). Combined with our previous finding that S3I-201 inhibited the endogenous expression of VEGF and TGF $\beta 1$, we conclude that S3I-201 inhibits both the fibrogenesis induced by TGF $\beta 1$ and angiogenesis by VEGF as well as the cross-talk between TGF $\beta 1$ and VEGF.

\section{Synergistic interaction between S3I-201 and sorafenib in LX-2 cells}

Sorafenib is a multikinase inhibitor approved by United States Food and Drug Administration for treatment of
Fluorescent conjugates of phalloidin were used to label actin filaments. Transformation of cell shape and disruption of actin fibers were observed by fluorescence microscope after $24 \mathrm{~h}$ in the presence of S3I-201. d S3I-201 inhibited the migration of H-HSC. H-HSC was cultured to confluence, disrupted to generate a linear wound and then incubated with/without S3I-201 for $20 \mathrm{~h}$. And would healing was photographed. Experiments were carried out in duplicate, and five fields of well were recorded

advanced liver cancer. To examine whether sorafenib can enhance the antifibrotic and anti-angiogenetic effects of S3I-201 on LX-2 cells, the dose-effect curves were measured for individual compounds and also the combination of these two compounds. To determine whether the combined treatment had a synergistic impact on cell proliferation, cells were exposed to the combined drug treatment in sequential dosages with a fix ratio (sorafenib: S3I-201 $=1: 5$ ) for $48 \mathrm{~h}$ and the combination index values for each dose were calculated. As shown in the combination index plot, at most combination doses, the combination index values were considerably less than 1.0 , indicating a synergistic interaction (Fig. 8a). The combination treatment greatly decreased the expression levels of collagen I, $\alpha$-SMA and TIMP-1 compared with S3I-201 or sorafenib monotherapy (Fig. 8b), indicating that cotreatment enhanced the antifibrotic effect on LX-2 cells. Similarly, the combination treatment dramatically reduced the expression of angiogenic molecules (VEGF, VEGFR1 and VEGFR2) and antiapoptotic molecules (Bcl-2 and $\mathrm{Bcl}-\mathrm{x}_{\mathrm{L}}$ ). Moreover, combination treatment also suppressed the expression of TGF $\beta$ RII significantly (Fig. 8b). 
A

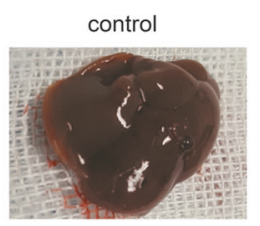

CCI4 2weeks

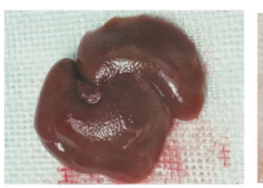

B
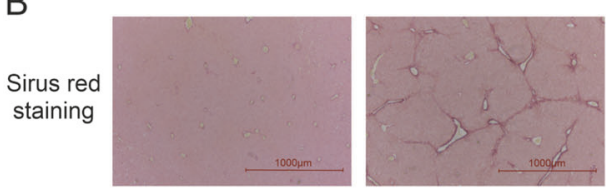

C
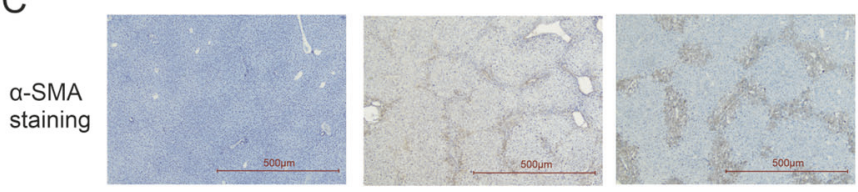

CCl4 2weeks+

D

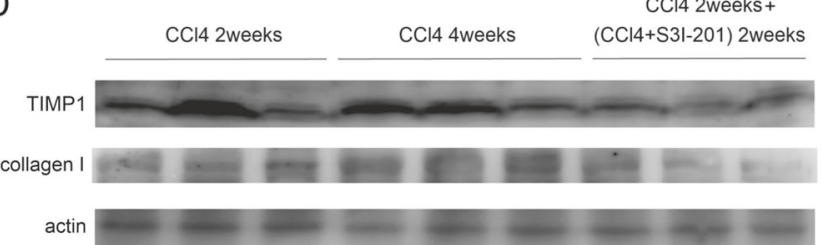

$\mathrm{CCl} 4$ weeks
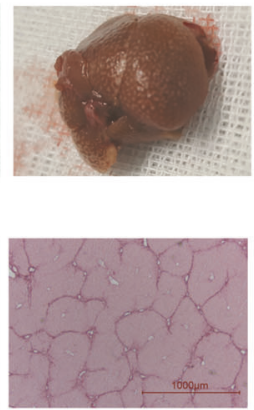

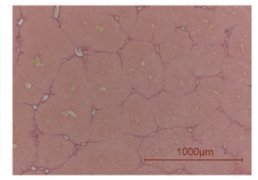

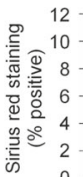

0 control CCl4 2wks CCl4 4wks CCl4 2wks $+(\mathrm{CCl} 4+\mathrm{S} 3 \mid-201)$
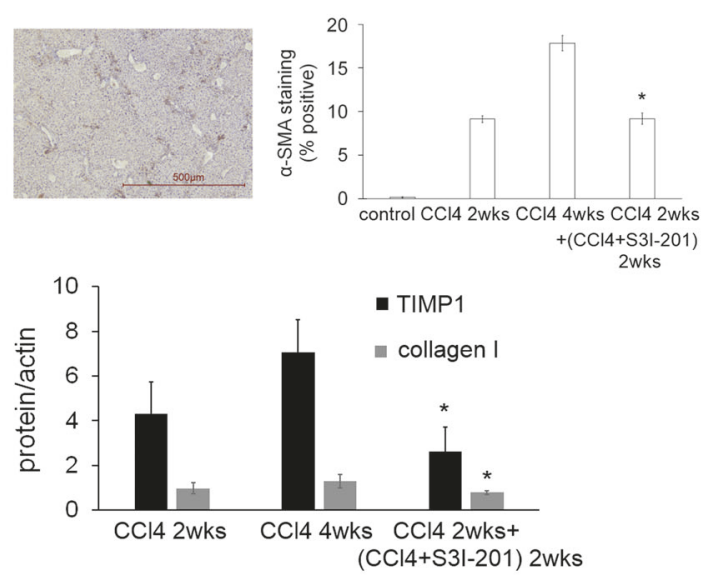

E
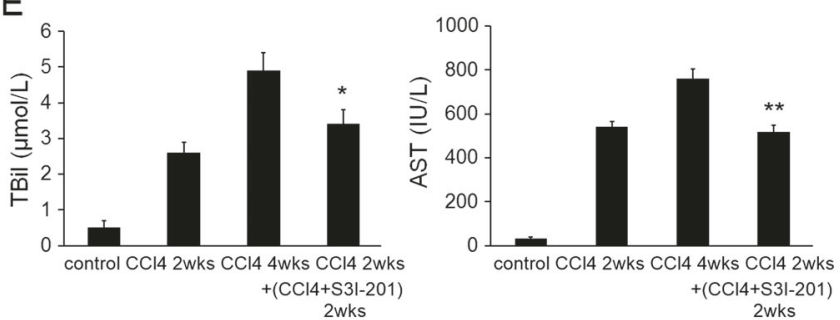

Fig. $3 \mathrm{~S} 3 \mathrm{I}-201$ inhibited the fibrosis in vivo. a $\mathrm{CCl}_{4}$-induced liver fibrosis model. Liver fibrosis was apparent by 2 weeks of $\mathrm{CCl}_{4}$ induction and was more severe after 4 weeks whereas S3I-201 significantly suppressed the progression of liver fibrosis. b S3I-201 significantly attenuated the collagen expression. Sirius red staining was used to reveal collagen deposition in liver. Collagen was evident after 2 weeks of $\mathrm{CCl}_{4}$ induction and was even more severe after 4 weeks. S3I-201 treatment greatly reduced collagen deposition. c Hepatic

\section{Discussion}

Initially developed as an anti-cancer drug, S3I-201 is more potent in liver fibrosis than in hepatocellular carcinoma [20], with an IC50 of $100 \mu \mathrm{M}$ in HCC while $40 \mu \mathrm{M}$ in HHSC (Fig. 2a). S3I-201 selectively inhibits the phosphorylation and dimerization of STAT3 [16], and thereafter the transcription of target genes controlled by STAT3. Our study confirmed that S3I-201 inhibited the production of p-STAT3 and suppressed the translocation of p-STAT3

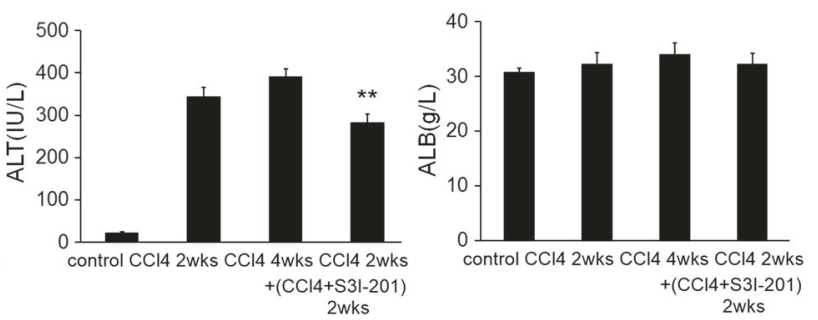

immunohistochemical staining with $\alpha$-SMA. Lots of $\alpha$-SMA-positive HSCs can be seen in livers of $\mathrm{CCl}_{4}$-induced mice, whereas significant reduction of $\alpha$-SMA-positive cells is observed in livers of S3I-201treatd mice. d Expression of collagen I and TIMP-1 in liver. Liver tissue lysate was analyzed for the expression of collagen I and TIMP1 by Western blotting. Representative results of three experiments were shown. e Levels of serum fibrosis markers, TBil, AST, ALT, and ALB, were examined using standard laboratory assays

from cytosol to nucleus but had no effect on the expression of total STAT3 (Fig. 1c).

Inhibition of HSC activation is crucial in antifibrotic therapies because activated HSCs are the major source of ECM proteins for liver fibrosis. STAT3 is suggested to play a protective role in attenuating liver fibrosis due to its hepatoprotective and proliferative functions in hepatocytes [21-25] while a counteractive role in HSCs where inhibition of the STAT3 pathway induces apoptosis of HSC [26]. Therefore, the antifibrotic potential of S3I-201 was first 

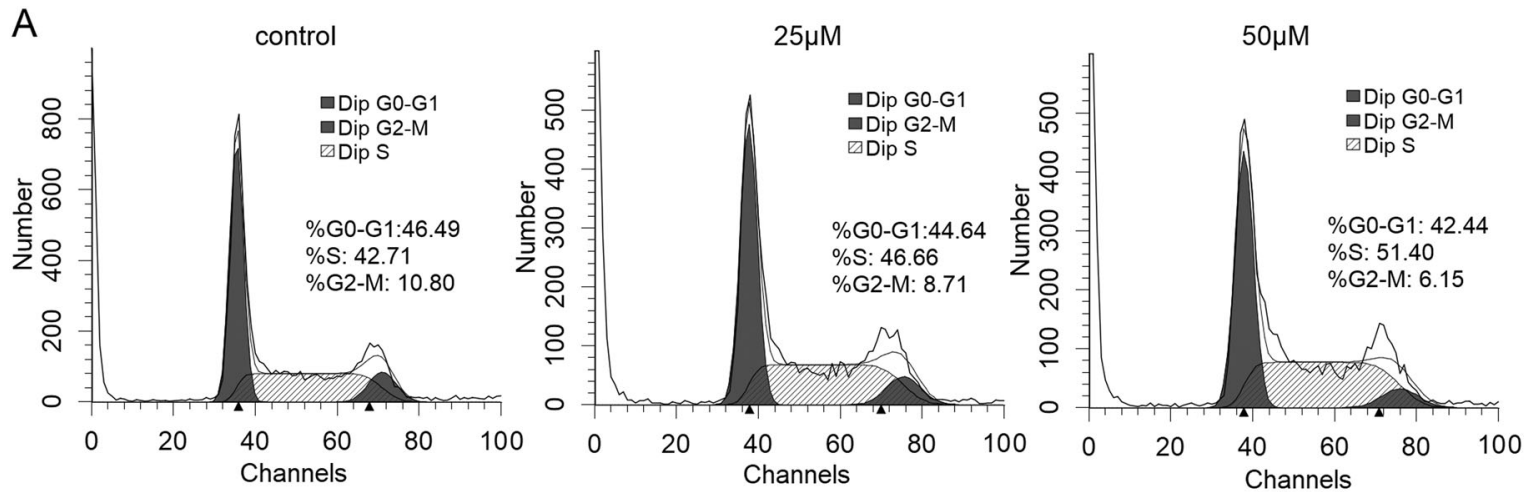

B

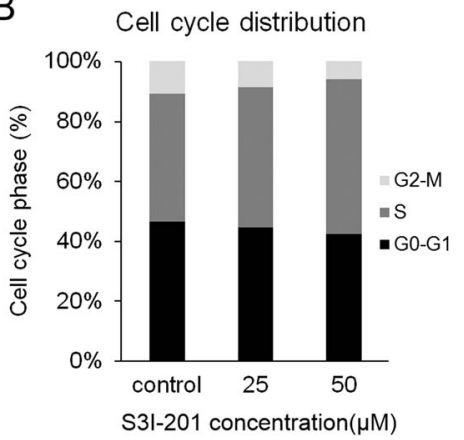

D

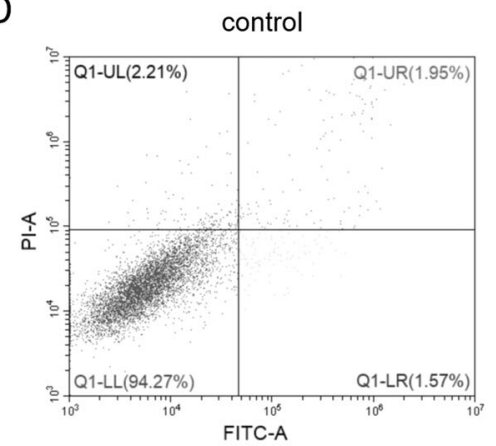

E

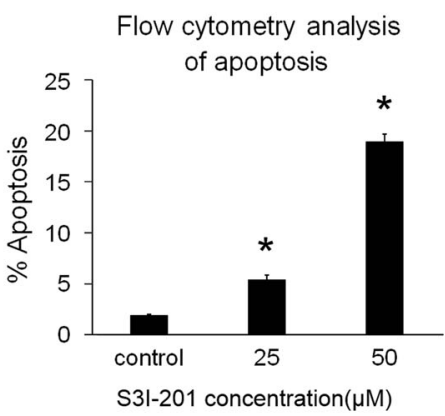

C
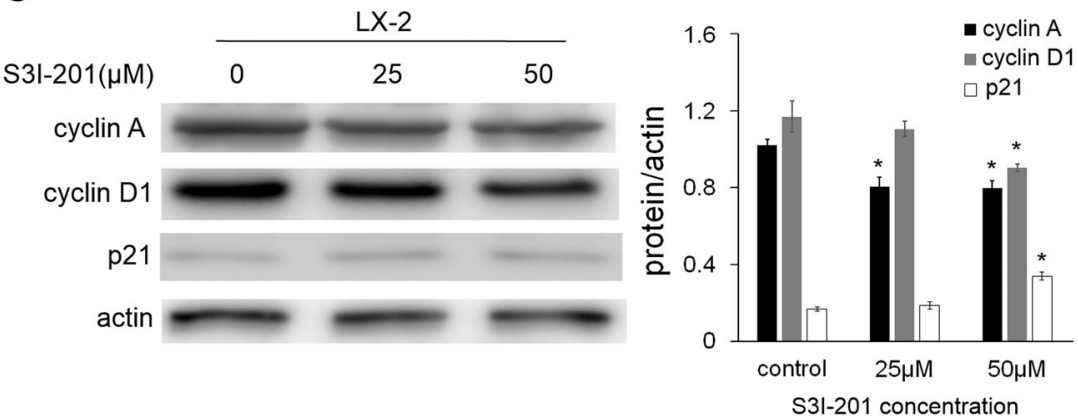

$25 \mu \mathrm{M}$

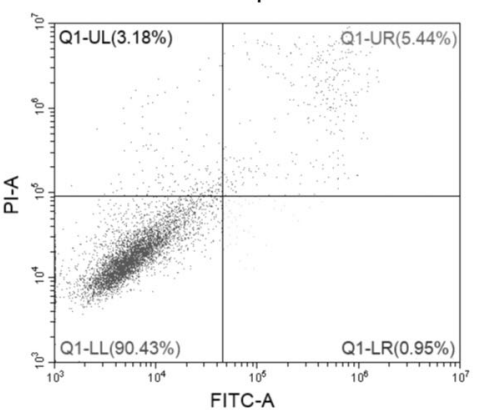

$\mathrm{F}$
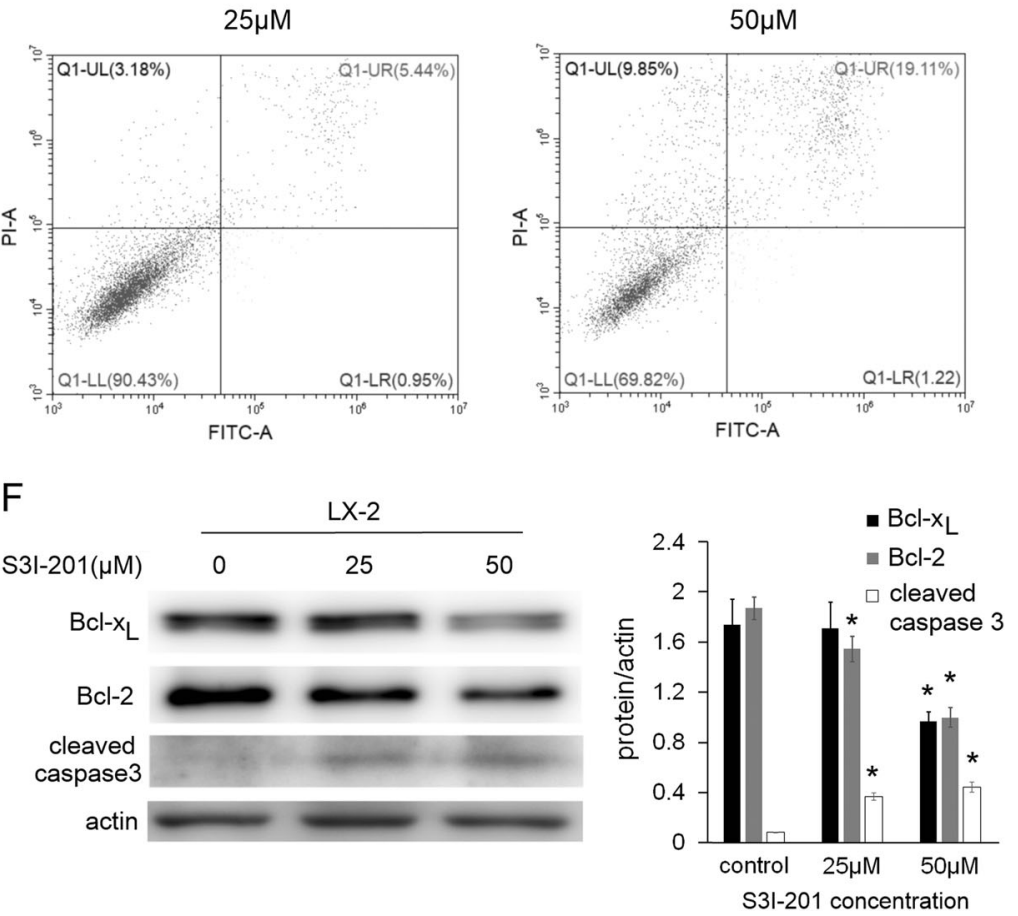

Fig. 4 S3I-201 induced cell cycle arrest and apoptosis in LX-2 cells. a, b Flow-cytometry analysis of LX-2 cells treated by S3I-201. LX-2 cells were incubated with S3I-201 for $24 \mathrm{~h}$ and flow cytometry analysis revealed that $25 \mu \mathrm{M}$ and $50 \mu \mathrm{M}$ of S3I-201 led to accumulation of cells in the S phase. c The effect of S3I-201 on the expression of cyclin A, cyclin D1 and p21 in LX-2. Cells were treated with S3I-201 for $24 \mathrm{~h}$, and cell lysates were analyzed for the expression of cyclin A, cyclin D1 and P21 by Western blotting. Representative result of three experiments were shown. d, e Flow cytometry analysis of S3I-201 treated LX-2 cells. LX-2 cells were treated with S3I-201 for $24 \mathrm{~h}$, and then harvested and double-stained with Annexin V-FITC and PI. Apoptosis was quantified as Annexin V positive and PI negative. Data were expressed as mean \pm SEM, $* p<0.05$. f Protein expression levels of cleaved caspase 3, Bcl-2 and Bcl- $\mathrm{x}_{\mathrm{L}}$ were analyzed by Western blotting. S3I-201 upregulated cleaved caspase 3 and downregulated Bcl-2 and $\mathrm{Bcl}-\mathrm{x}_{\mathrm{L}}$. Representative result of three experiments was shown 
A

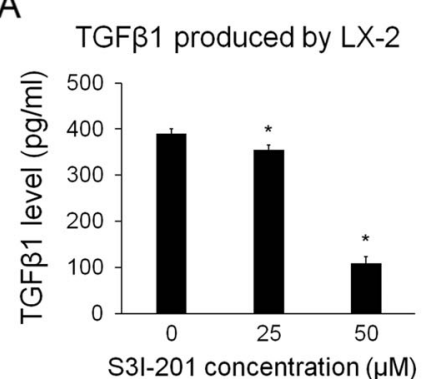

B
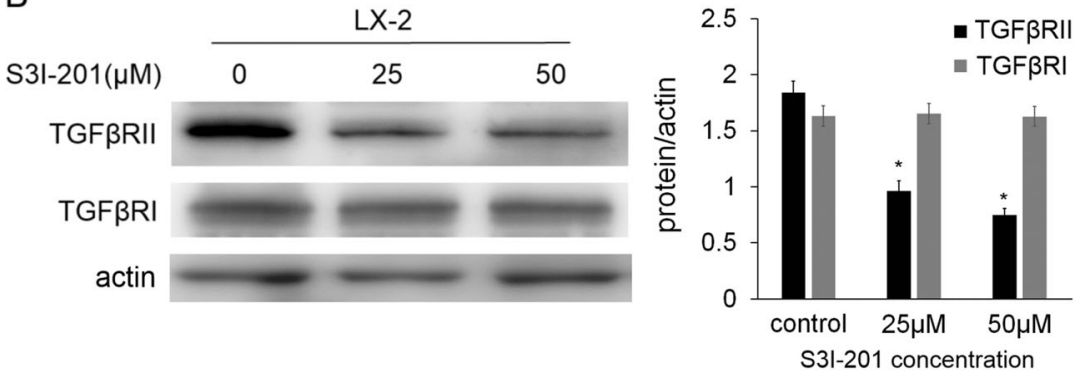
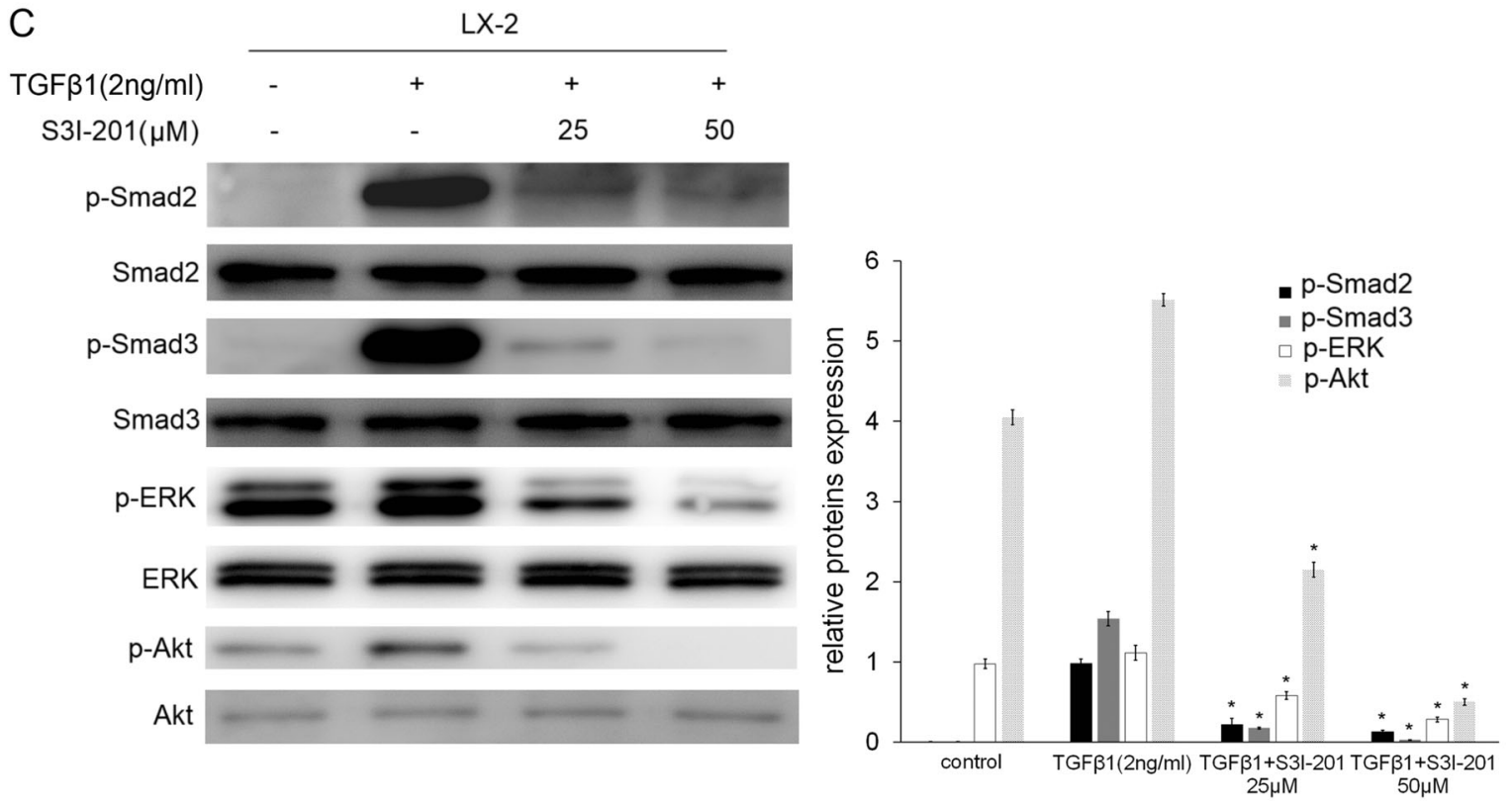

$\mathrm{D}$
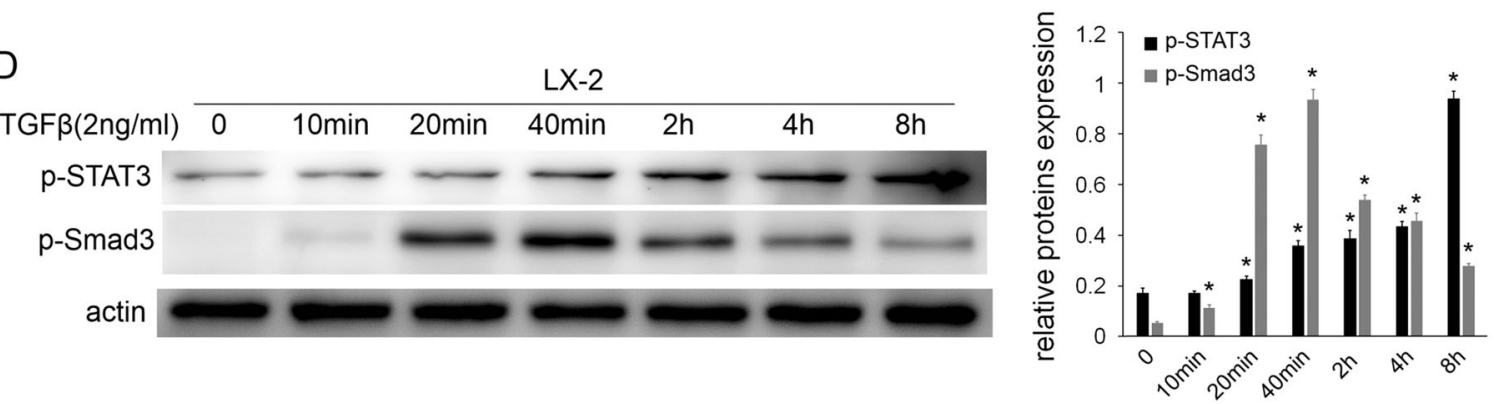

Fig. 5 S3I-201 inhibited the secretion of TGF $\beta 1$ and expression of TGFßRII, as well as TGF $\beta 1$-induced phosphor-Smad2, -Smad3, -Akt and -ERK. a Secretory expression of TGF $\beta 1$ in LX-2 upon S3I-201 treatment. LX-2 were cultured in different doses of S3I-201 for $24 \mathrm{~h}$. The culture supernatants were collected and analyzed for TGF $\beta 1$ level using ELISA kit. Data were expressed as mean \pm SEM., $* p<0.05$. b S3I-201 suppressed the expression of TGF $\beta$ RII but not TGF $\beta$ RI. LX-2 cells were treated with S3I-201 for $24 \mathrm{~h}$, and the whole lysates

evaluated in vitro on primary HSC. S3I-201 inhibited the activation of H-HSC by suppressing its proliferation, migration, stress fiber formation, as well as the expression of fibrosis-promoting factors $\alpha$-SMA, collagen and TIMP1 (Fig. 2). Furthermore, the antifibrotic effect of S3I-201 was also observed in vivo in $\mathrm{CCl}_{4}$-induced fibrotic mouse were collected and analyzed by Western blotting. c S3I-201 inhibited TGFß1-induced phosphorylation of Smad2, Smad3, Akt and ERK. S3I-201 was added to LX-2 cells for $6 \mathrm{~h}$ prior to TGF $\beta 1(2 \mathrm{ng} / \mathrm{ml})$ stimulation for $20 \mathrm{~min}$. The expression levels of p-STAT3, p-Smad2, p-Smad3, p-ERK and p-Akt were analyzed by Western blotting. d LX2 cells were starved overnight and treated with $\operatorname{TGF} \beta(2 \mathrm{ng} / \mathrm{ml})$ at different times, and the expression of p-STAT3 and p-Smad3 was examined by Western blotting

model, which is evidenced by the downregulation of collagen deposition, $\alpha$-SMA and TIMP-1 expression (Fig. 3).

S3I-201 treatment not only significantly suppressed the activation but also inhibited the proliferation of HSC (Fig. 2a). The antiproliferative effect of S3I-201 on HSC shows to be mediated by multiple mechanisms. On one 
A

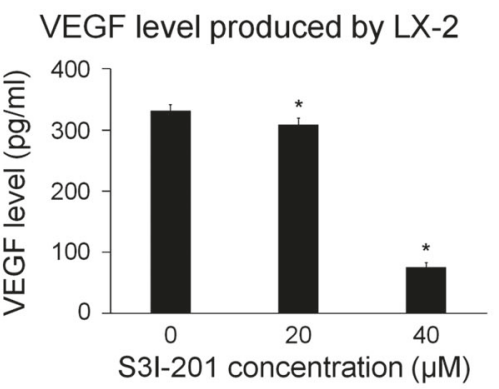

B

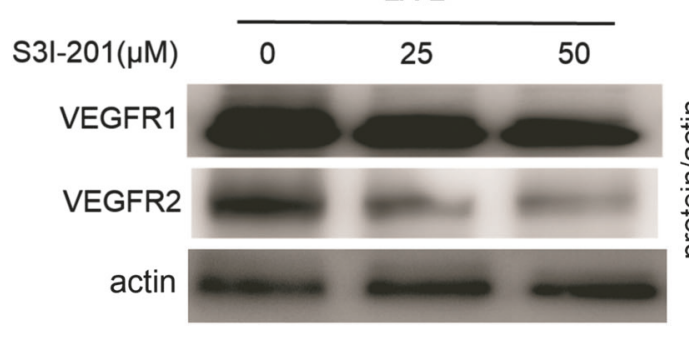

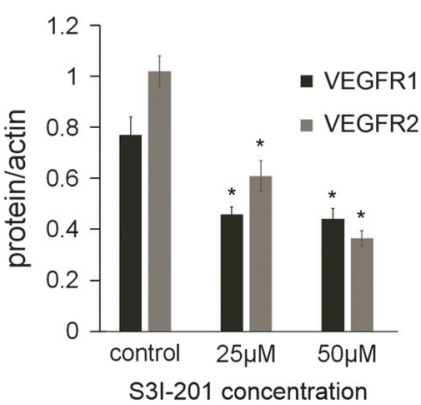

C

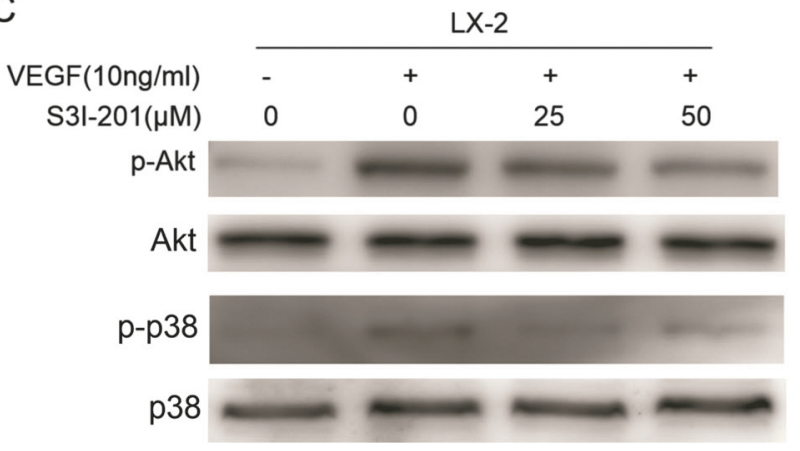

D

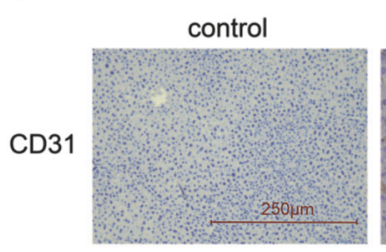

CCl4 2weeks

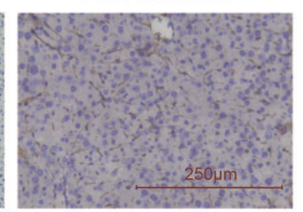

$\mathrm{CCl} 4$ 4weeks

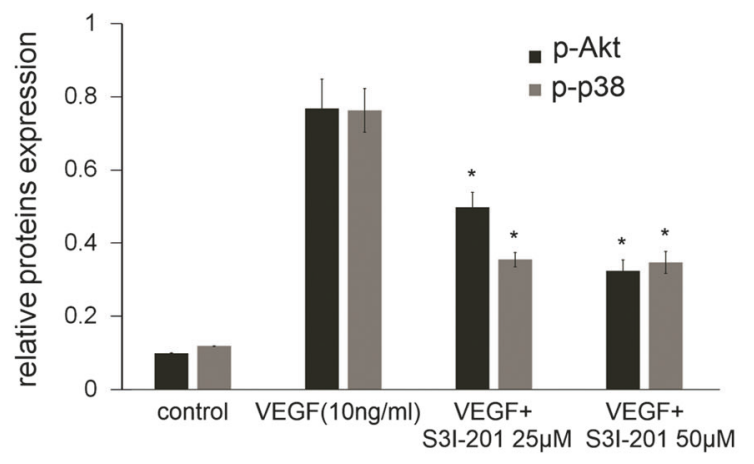

E
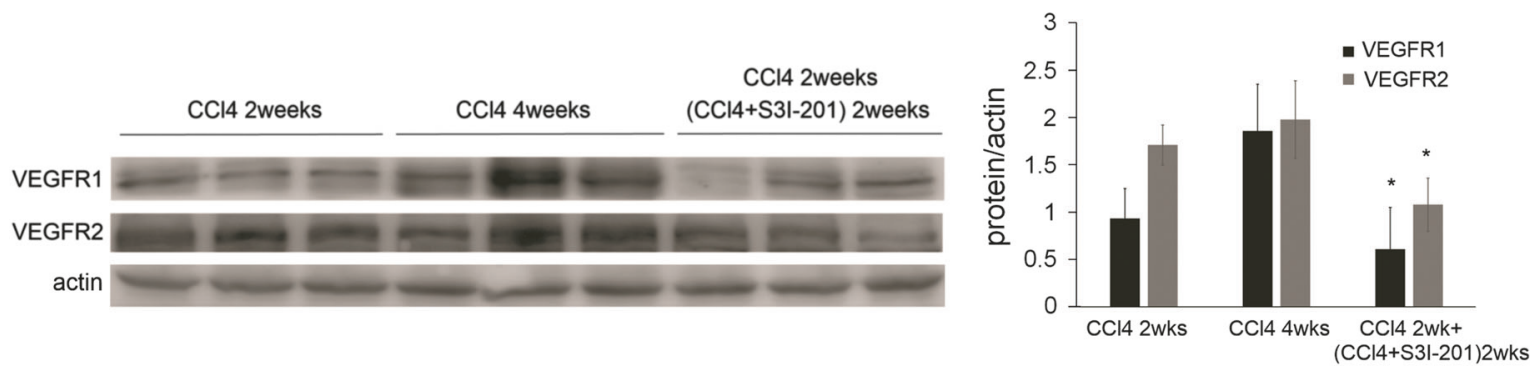

Fig. 6 S3I-201 inhibited the angiogenesis both in vitro and in vivo. a S3I-201 treatment inhibited the secretory expression of VEGF in LX-2. LX-2 cells were cultured in different doses of S3I-201 for $24 \mathrm{~h}$. The culture supernatants were collected and analyzed for VEGF level using ELISA kit. Data were expressed as mean \pm SEM., $* p<0.05$. b S3I-201 suppressed the expression of VEGFR1 and VEGFR2. LX-2 cells were treated with S3I-201 for $24 \mathrm{~h}$, and the whole lysates were collected and analyzed for the expression levels of VEGFR1 and VEGFRII by Western blotting. c S3I-201 inhibited VEGF-induced Akt

side, S3I-201 induced growth arrest of HSC with more cells in the $\mathrm{S}$ phase. This effect was associated with an increase in the expression of p21 and decrease in cyclin A and cyclin D1 (Fig. 4). On the other side, S3I-201 induced apoptosis of

and p38 MAPK phosphorylation. S3I-201 was added to LX-2 for $6 \mathrm{~h}$ prior to VEGF $(10 \mathrm{ng} / \mathrm{ml})$ stimulation for $20 \mathrm{~min}$. The expression of $\mathrm{p}$ Akt and p-p38 was analyzed by Western blotting. d Immunohistochemical staining of CD31 in liver section treated with or without S3I201. Abundant microvessels stained positively with CD31 were observed in fibrotic septa (brown color, original magnification $\times 200$ ). e S3I-201 treatment significantly decreased VEGFR1 and VEGFR2 expression in liver tissue as analyzed by Western blotting. Data were representative of three experiments

HSCs (Fig. 4), which is an important mechanism for liver fibrosis resolution by reducing the number of activated HSCs. S3I-201 treatment increased the cleavage of caspase3, a central caspase in the apoptotic cascade [27], and 
$\mathrm{A} 1$

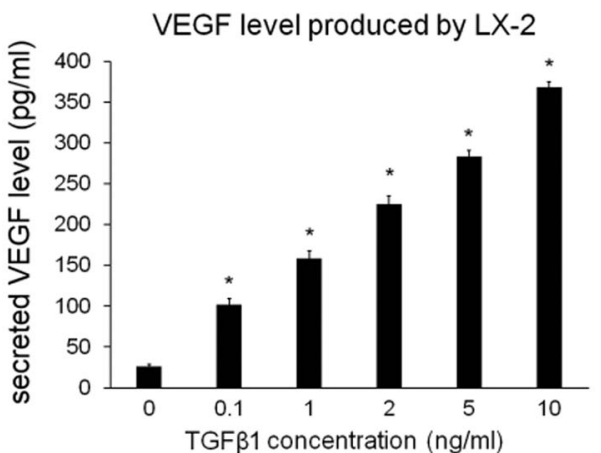

B1

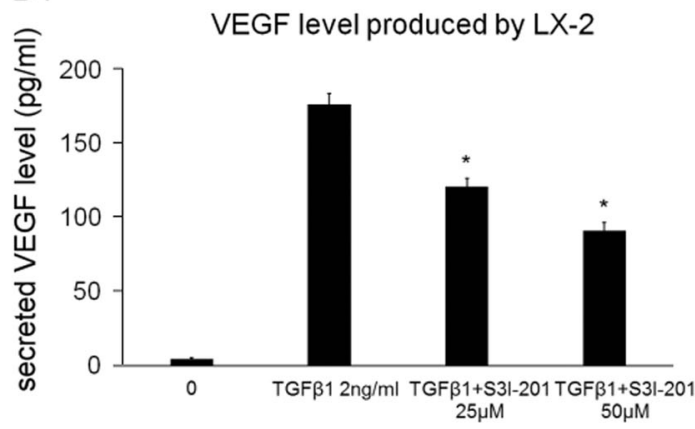

A2 LX-2

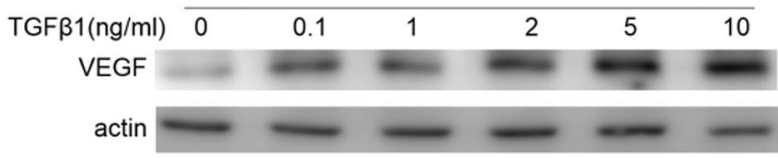

B2

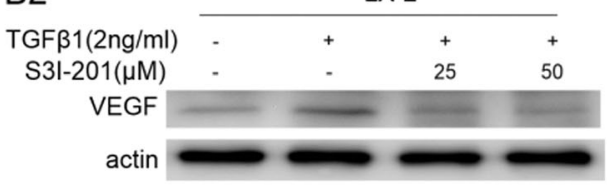

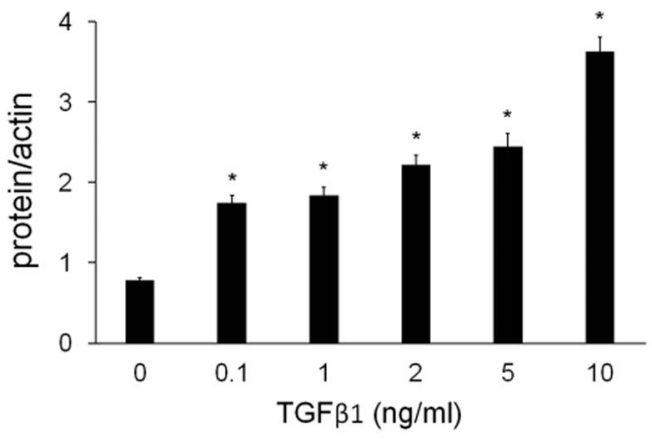

Fig. 7 S3I-201 attenuated TGF $\beta$-induced upregulation of VEGF in vitro. (A1) and (A2) TGF $\beta$ upregulated the expression of VEGF in LX-2 cells. LX-2 cells were starved overnight and exposed to different doses of TGF $\beta 1$ for $6 \mathrm{~h}$. The medium supernatant was collected and analyzed by ELISA assay (A1), and the cell lysates were analyzed by

decreased the expression of Bcl-2 and $\mathrm{Bcl}-\mathrm{x}_{\mathrm{L}}$, the overexpression of which contributes to the development of fibrosis by enhancing the resistance to apoptosis in activated HSCs [28].

In response to liver injury, activated HSC secretes TGF $\beta$, which triggers an autocrine positive feedback loop inducing fibrogenesis via SMAD2/SMAD3. The function of TGF $\beta 1$ is cell-type-dependent, and might even be opposing at different liver disease stages [29]. TGF $\beta 1$ plays a critical role in controlling liver mass via regulating the cytostasis and apoptosis of hepatocytes in liver development and regeneration. Therefore, loss of TGF $\beta 1$ activation could lead to hyperproliferative disorders and cancer. Whereas, high level of TGF $\beta 1$, as a consequence of liver injury, activates HSCs, promotes myofibroblast transdifferentiation, stimulates production of extracellular matrix and thus is a pivotal profibrogenic cytokine [9]. Furthermore, TGF $\beta 1$ is reported to be a new target in the list of STAT3-regulated genes involved in fibrogenesis [11]. Our study demonstrated that STAT3 inhibitor S3I-201 could reduce the expression of

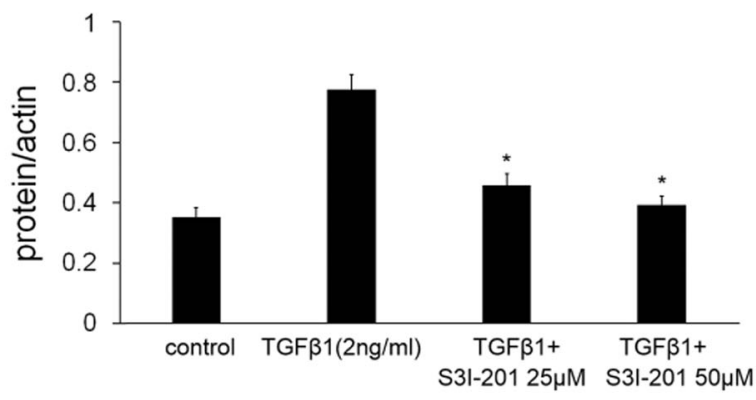

Western blotting (A2). (B1) and (B2) S3I-201 inhibited the expression of VEGF induced by TGF $\beta$. S3I-201 was added to LX-2 for $6 \mathrm{~h}$ prior to TGF $\beta 1(2 \mathrm{ng} / \mathrm{ml})$ stimulation for $6 \mathrm{~h}$. The supernatant (B1) and cell lysate (B2) were collected and analyzed by ELISA and western blot, respectively

TGF $\beta 1$ (Fig. 5a), consolidating TGF $\beta 1$ as a STAT3 target gene. Additionally, S3I-201 inhibited the expression of TGFßRII significantly but not TGF $\beta$ RI (Fig. 5b), implicating that STAT3 inhibition could attenuate TGF $\beta$-induced canonical pathway in HSC (Fig. 5c). In addition, Akt and Erk involved non-canonical TGF $\beta 1$ signaling pathway that mediated the survival, proliferation, apoptosis resistance and collagen production of HSCs [30, 31], was also inhibited by S3I-201 (Fig. 5c). In one word, through inhibition of STAT3, S3I-201 downregulated the expression levels of TGF $\beta 1$ and TGF $\beta$ RII and thereafter both canonical and non-canonical pathways mediated by TGF $\beta 1$, resulting attenuation of HSC activation.

The signaling of TGF $\beta$ family is mediated by TGF $\beta$ RII, which recruits TGF $\beta$ RI and then phosphorylates receptoractivated Smad. As the best-characterized TGF $\beta$-response intracellular effectors, Smad signaling from cell surface to nucleus has been well-established [32]. However, the crosstalk between STAT3 and TGF $31 /$ Smad signaling in HSC and fibrogenesis has not been elucidated clearly. To date, 
A
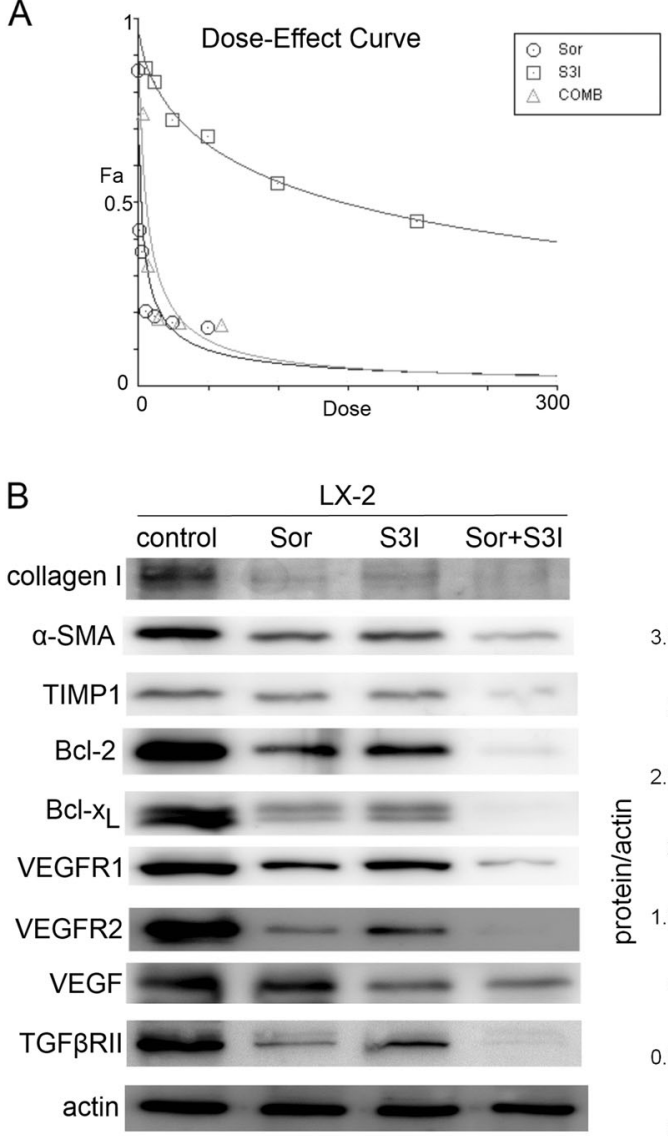

Fig. 8 Synergistic effect of S3I-201 and sorafenib in LX-2 cells. a Dose-effect and combination index of sorafenib and S3I-201. Cells were treated with sequential concentration of drugs for $48 \mathrm{~h}$ and the IC50 values were calculated. To determine whether the combined treatment had a synergistic effect on cell proliferation, the combination index values of each dose were calculated by the CompuSyn software. Cells were exposed to the combined drug treatment in sequential dosages with a fix ratio (sorafenib: S3I-201=1: 5) for $48 \mathrm{~h}$. The

there are several reports, albeit conflicting, demonstrating crosstalk between TGF $\beta 1$ and STAT3 signaling pathways. Tang et al. reported a cooperative role of STAT3 with TGF $\beta 1 /$ Smad3 [9]. Wang et al illustrated a physical direct interaction of STAT3 with $\operatorname{Smad} 3$ attenuated TGF $\beta 1$ induced response [33]. In this study, we observed that TGF $\beta 1$ did induce the phosphorylation of Smad2 and Smad3 obviously while STAT3 inhibition could reduce this effect dramatically, implicating that STAT3 might cooperate Smad $2 / 3$ activation in early phase $(20 \mathrm{~min})$ of TGF 11 stimulation. We also determined the levels of p-STAT3 and p-Smad3 stimulated by TGF $\beta 1$ over the time course of several hours. Interestingly, the data showed that p-STAT3 increased in a time-dependent manner. Whereas, two phases of $\mathrm{p}$-Smad3 responses were observed with an initial activation peaking at $40 \mathrm{~min}$ (Fig. 5d). The cooperation between STAT3 and Smad3 sustained 40 min upon
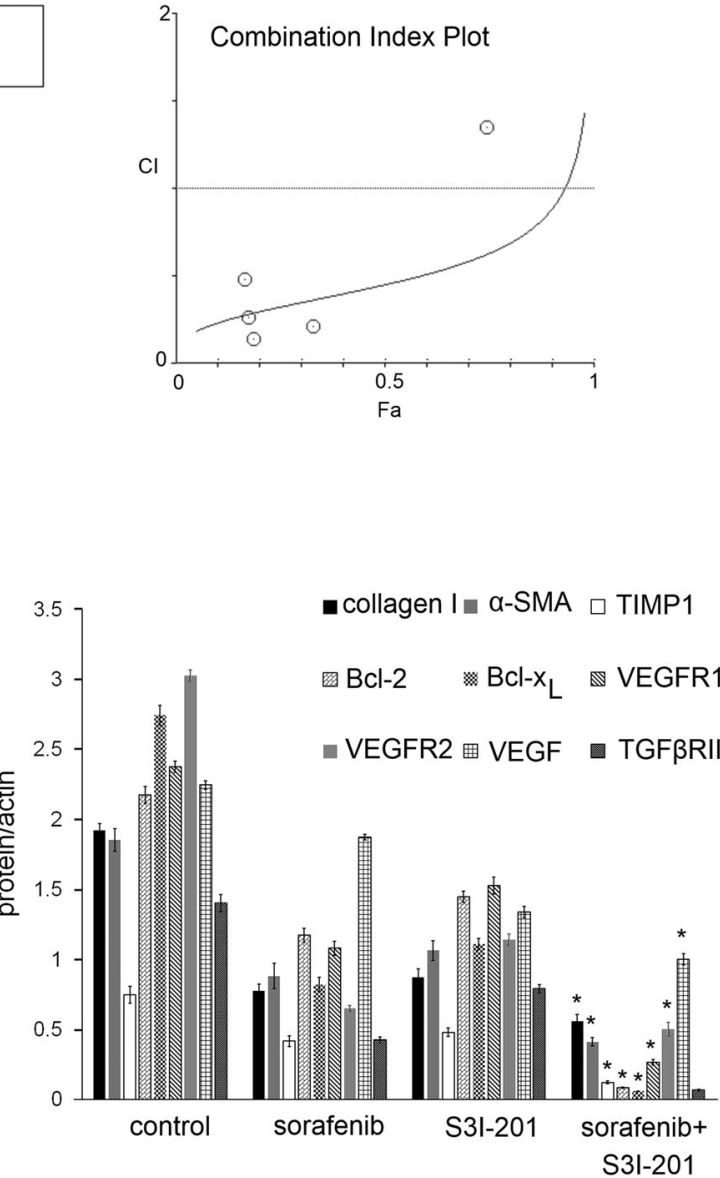

proliferation rate was assessed by MTS assay. b Combination treatment significantly decreased the expression of pro-fibrotic ECM factors collagen I, $\alpha$-SMA and TIMP-1, anti-apoptotic factors Bcl-2 and $\mathrm{Bcl}-\mathrm{x}_{\mathrm{L}}$, angiogenetic factors VEGF, VEGFRI and VEGFRII and pro-fibrotic factor TGF $\beta$ RI and TGF $\beta$ RII compared with S3I-201 or sorafenib treatment. LX-2 cells were treated with sorafenib, S3I-201, and sorafenib $+\mathrm{S} 3 \mathrm{I}-201$ for $24 \mathrm{~h}$, the protein expression levels were analyzed by Western blotting

TGF $\beta 1$ stimulation and p-Smad3 decreased later, which might be due to association of Smad3 with Smad4 and nucleus translocation, and more detailed mechanism needs to be investigated further.

Angiogenesis is believed to promote fibrosis, largely due to the observation that the changes of hepatic sinusoidal endothelial cells and neovascularization occur simultaneously during the development of liver fibrosis [34]. HSCs exert a pro-angiogenic role in fibrosis through promoting the expression VEGF and VEGFR [14]. Overexpressed VEGF also accelerates intestinal inflammation [35] and promote liver fibrosis through regulating monocyte infiltration [36] and HSC activation [37]. VEGF binds two tyrosine kinase receptors VEGFR1 and VEGFR2 with VEGFR2 as the major mediator and VEGFR1 as a decoy, which regulates angiogenesis and microvascular permeability. [38]. Our results showed that S3I-201 inhibited not 
only the expression of VEGFR1 and VEGFR2 but also the secretion of VEGF by LX-2 cells (Fig. 6a, b), indicating that S3I-201 may disrupt VEGF autocrine signaling. VEGF induced both the proliferation and procollagen synthesis in activated HSC [14] possibly through p-Akt, an important intracellular mediator which stimulates HSC proliferation, promotes resistance to apoptosis and collagen deposition $[30,31]$. Furthermore, it is reported that VEGF-induced VEGFR-dependent p38MAPK activation is required for tubulogenesis in human endothelial cells [39] and that p38MAPK activation associated with increased collagen production in activated HSC [40]. Our data demonstrate that S3I-201 inhibited VEGF-induced upregulation of p-Akt and p38MAPK (Fig. 6c), and hence the angiogenesis of HSCs. Furthermore, we evaluated the anti-angiogenesis effect of S3I-201 in vivo using $\mathrm{CCl}_{4}$-induced liver fibrosis mouse model. CD31, a neovascularization marker, was stained to demonstrate the formation of microvessels. In fibrotic livers, CD31 positive cells were observed while S3I-201 treatment significantly reduced the number of CD31positive cells (Fig. 6d). Moreover, similar to in vitro assays, the expression levels of VEGFR1 and VEGFR2 also decreased in S3I-201-treated fibrotic liver (Fig. 6e). Both the in vitro and in vivo results show that S3I-201 treatment could inhibit VEGF-induced angiogenesis in liver fibrosis.

Consistent with the result obtained in mouse HSCs [41], our data confirm that TGF $\beta 1$ induced VEGF expression in H-HSC in both total and secreted forms. STAT3 inhibition decreases the expression levels of both TGF $\beta 1$ (Fig. 5a) and VEGF (Fig. 6a), which was also observed in other studies $[42,43]$. In addition to the individual inhibitory effects of S3I-201 on the expression of VEGF and TGF $\beta 1$, our data revealed the inhibitory effect of S3I-201 on crosstalk between TGF $\beta 1$ and VEGF, showing that S3I-201 inhibited the elevated level of VEGF autocrine induced by TGF $\beta 1$ (Fig. 7).

Sorafenib is a multikinase inhibitor used for the treatment of advanced HCC, which blocks the phosphorylation of several kinases including Raf, VEGFR, and platelet-derived growth factor receptor [44]. Recently, sorafenib is also applied in liver fibrosis treatment [45]. However, sorafenibinduced apoptosis was abolished in STAT3-overexpressing HSC cells, indicating that STAT3 may be a key player in sorafenib-induced apoptosis [46]. The level of hepatic p-STAT3 decreased in early anti-fibrotic treatment by sorafenib but soon increased in response to dynamic regulation of IL-6 signaling in Kupffer cells [22]. Therefore, combinational therapy by co-targeting receptor tyrosine kinases and STAT3 pathway provides a promising strategy to improve clinical prognosis.

To date, no effective therapy against STAT3 has been approved despite the fact that abundant evidence support STAT3 as a potential anti-cancer target. The lack of efficacious therapeutics is largely due to its numerous activators. However, S3I-201, as a direct inhibitor of STAT3, overcomes this barrier. In this project, we explored the effect of sorafenib and S3I-201 combination treatment in liver fibrosis. Using the chou and Talalay method, we evaluated the interaction between sorafenib and S3I-201 through combination treatment on LX-2 cells. Our data clearly showed that sorafenib and S3I-201 inhibited liver fibrosis highly synergistically in multiple mechanisms, including downregulating the expression of pro-fibrotic ECM factors collagen I, $\alpha$-SMA and TIMP1, inducing apoptosis, suppressing the expression of pro-angiogenetic factors VEGF and VEGFR as well as decreasing the expression of pro-fibrotic signal TGFßRII. The synergistic interaction between sorafenib and S3I-201 would potentiate the combination therapy in liver fibrosis and deserves further characterization.

In conclusion, our in vitro and in vivo results demonstrated that STAT3 inhibitor S3I-201 attenuated the development of liver fibrosis markedly through multiple mechanisms including induction of HSC apoptosis and cell cycle arrest, inhibition of TGF $\beta$ signaling pathway, as well as suppression of neo-angiogenesis. Therefore, our study underscores the rationale for the further development of STAT3 inhibitors alone or in combination with sorafenib for patients with liver fibrosis.

Acknowledgements This work was partly supported by Guangdong Science and Technology Program (2017B030301018), research grants from Shenzhen Science and Technology Innovation Committee (JCYJ20160608140912962 and ZDSYS20140509142721429) and National Natural Science Foundation of China (31670753).

Conflict of Interest The authors declare no conflict of interest.

\section{References}

1. Bataller R, Brenner DA. Liver fibrosis. J Clin Invest. 2005;115:209-18.

2. Higashi T, Friedman SL, Hoshida Y. Hepatic stellate cells as key target in liver fibrosis. Adv Drug Deliv Rev. 2017;121:27-42.

3. Trautwein C, Friedman SL, Schuppan D, et al. Hepatic fibrosis: concept to treatment. J Hepatol. 2015;62:15-24.

4. Lee YA, Wallace MC, Friedman SL. Pathobiology of liver fibrosis: a translational success story. Gut. 2015;64:830-41.

5. Ellis EL, Mann DA. Clinical evidence for the regression of liver fibrosis (vol 56, pg 1171, 2012). J Hepatol. 2014;60:468-9.

6. Wasmuth HE, Tacke F, Trautwein C. Chemokines in liver inflammation and fibrosis. Semin Liver Dis. 2010;30:215-25.

7. Gressner AM, Weiskirchen R, Breitkopf K, et al. Roles of TGFbeta in hepatic fibrosis. Front Biosci. 2002;7:793-807.

8. Dooley S, Hamzavi J, Breitkopf K, et al. Smad7 prevents activation of hepatic stellate cells and liver fibrosis in rats. Gastroenterology. 2003;125:178-91.

9. Tang LY, Heller M, Meng Z, et al. Transforming growth factorbeta (TGF-beta) directly activates the JAK1-STAT3 axis to induce hepatic fibrosis in coordination with the SMAD pathway. J Biol Chem. 2017;292:4302-12. 
10. Liu XJ, Hu H, Yin JQ. Therapeutic strategies against TGF-beta signaling pathway in hepatic fibrosis. Liver Int. 2006;26:8-22.

11. Xu MY, Hu JJ, Shen J, et al. Stat3 signaling activation crosslinking of TGF-beta 1 in hepatic stellate cell exacerbates liver injury and fibrosis. Bba-Mol Basis Dis. 2014;1842:2237-45.

12. Ogata H, Chinen T, Yoshida T, et al. Loss of SOCS3 in the liver promotes fibrosis by enhancing STAT3-mediated TGF-beta 1 production. Oncogene. 2006;25:2520-30.

13. Corpechot C, Barbu V, Wendum D, et al. Hypoxia-induced VEGF and collagen I expressions are associated with angiogenesis and fibrogenesis in experimental cirrhosis. Hepatology. 2002;35: 1010-21.

14. Yoshiji H, Kuriyama S, Yoshii J, et al. Vascular endothelial growth factor and receptor interaction is a prerequisite for murine hepatic fibrogenesis. Gut. 2003;52:1347-54.

15. Alvarez JV, Febbo PG, Ramaswamy S, et al. Identification of a genetic signature of activated signal transducer and activator of transcription 3 in human tumors. Cancer Res. 2005;65:5054-62.

16. Siddiquee K, Zhang S, Guida WC, et al. Selective chemical probe inhibitor of Stat3, identified through structure-based virtual screening, induces antitumor activity. Proc Natl Acad Sci USA. 2007;104:7391-6.

17. Liu YQ, Wang Z, Wang JN, et al. A histone deacetylase inhibitor, largazole, decreases liver fibrosis and angiogenesis by inhibiting transforming growth factor-beta and vascular endothelial growth factor signalling. Liver Int. 2013;33:504-15.

18. Liu YQ, Wang Z, Kwong SQ, et al. Inhibition of PDGF, TGFbeta, and Abl signaling and reduction of liver fibrosis by the small molecule Bcr-Abl tyrosine kinase antagonist Nilotinib. J Hepatol. 2011;55:612-25.

19. Chou TC. Drug combination studies and their synergy quantification using the Chou-Talalay method. Cancer Res. 2010;70:440-6.

20. Lin L, Amin R, Gallicano GI, et al. The STAT3 inhibitor NSC 74859 is effective in hepatocellular cancers with disrupted TGFbeta signaling. Oncogene. 2009;28:961-72.

21. Horiguchi N, Lafdil F, Miller AM, et al. Dissociation between liver inflammation and hepatocellular damage induced by carbon tetrachloride in myeloid cell-specific signal transducer and activator of Transcription 3 gene knockout mice. Hepatology. 2010;51:1724-34.

22. Deng YR, Ma HD, Tsuneyama K, et al. STAT3-mediated attenuation of $\mathrm{CCl} 4$-induced mouse liver fibrosis by the protein kinase inhibitor sorafenib. J Autoimmun. 2013;46:25-34.

23. Mair M, Zollner G, Schneller D, et al. Signal transducer and activator of transcription 3 protects from liver injury and fibrosis in a mouse model of sclerosing cholangitis. Gastroenterology. 2010;138:2499-508.

24. Plum W, Tschaharganeh DF, Kroy DC, et al. Lack of glycoprotein 130/signal transducer and activator of transcription 3-mediated signaling in hepatocytes enhances chronic liver injury and fibrosis progression in a model of sclerosing cholangitis. Am J Pathol. 2010;176:2236-46.

25. Wang H, Lafdil F, Kong XN, et al. Signal transducer and activator of transcription 3 in liver diseases: a novel therapeutic target. Int $\mathbf{J}$ Biol Sci. 2011;7:536-50.

26. Jiang JX, Mikami K, Venugopal S, et al. Apoptotic body engulfment by hepatic stellate cells promotes their survival by the JAK/STAT and Akt/NF-kappa B-dependent pathways. J Hepatol. 2009;51:139-48.

27. Grutter MG. Caspases: key players in programmed cell death. Curr Opin Struct Biol. 2000;10:649-55.

28. Novo E, Marra F, Zamara E, et al. Overexpression of Bcl-2 by activated human hepatic stellate cells: resistance to apoptosis as a mechanism of progressive hepatic fibrogenesis in humans. Gut. 2006;55:1174-82.

29. Fabregat I, Moreno-Caceres J, Sanchez A, et al. TGF-beta signalling and liver disease. Febs J. 2016;283:2219-32.

30. Lechuga CG, Hernandez-Nazara ZH, Rosales JAD, et al. TGF-beta 1 modulates matrix metalloproteinase-13 expression in hepatic stellate cells by complex mechanisms involving p38MAPK, PI3-kinase, AKT, andp70(S6k). Am J Physiol Gastrointest Liver Physiol. 2004;287:974-87.

31. Reif S, Lang A, Lindquist JN, et al. The role of focal adhesion kinase-phosphatidylinositol 3-kinase-Akt signaling in hepatic stellate cell proliferation and type I collagen expression. J Biol Chem. 2003;278:8083-90.

32. Feng XH, Derynck R. Specificity and versatility in TGF-beta signaling through Smads. Annu Rev Cell Dev Bi. 2005;21: 659-93.

33. Wang G, Yu Y, Sun C, et al. STAT3 selectively interacts with Smad3 to antagonize TGF-beta signalling. Oncogene. 2016;35: 4388-98.

34. Lee JS, Semela D, Iredale J, et al. Sinusoidal remodeling and angiogenesis: a new function for the liver-specific pericyte? Hepatology. 2007;45:817-25.

35. Chen XH, Yang GX, Song JH, et al. Probiotic yeast inhibits VEGFR signaling and angiogenesis in intestinal inflammation. PLoS ONE. 2013;8:e64227.

36. Yang L, Kwon J, Popov Y, et al. Vascular endothelial growth factor promotes fibrosis resolution and repair in mice. Gastroenterology. 2014;146:1339-50.

37. Nakamura I, Zakharia K, Banini BA, et al. Brivanib attenuates hepatic fibrosis in vivo and stellate cell activation in vitro by inhibition of FGF, VEGF and PDGF Signaling. PLoS ONE. 2015; 10:e0142355.

38. Ferrara N, Gerber HP, LeCouter J. The biology of VEGF and its receptors. Nat Med. 2003;9:669-76.

39. Yang SY, Xin XH, Zlot C, et al. Vascular endothelial cell growth factor-driven endothelial tube formation is mediated by vascular endothelial cell growth factor receptor-2, a kinase insert domaincontaining receptor. Arter Throm Vas. 2001;21:1934-40.

40. Varela-Rey M, Montiel-Duarte C, Oses-Prieto JA, et al. p38 MAPK mediates the regulation of alpha $1(\mathrm{I})$ procollagen mRNA levels by TNF-alpha and TGF-beta in a cell line of rat hepatic stellate cells. FEBS Lett. 2002;528:133-8.

41. Liu YQ, Wen XM, Lui ELH, et al. Therapeutic targeting of the PDGF and TGF-beta-signaling pathways in hepatic stellate cells by PTK787/ZK22258. Lab Invest. 2009;89:1152-60.

42. Gurbuz V, Konac E, Varol N, et al. Effects of AG490 and S3I-201 on regulation of the JAK/STAT3 signaling pathway in relation to angiogenesis in TRAIL-resistant prostate cancer cells in vitro. Oncol Lett. 2014;7:755-63.

43. Pang MY, Ma L, Gong RJ, et al. A novel STAT3 inhibitor, S3I-201, attenuates renal interstitial fibroblast activation and interstitial fibrosis in obstructive nephropathy. Kidney Int. 2010;78:257-68.

44. Wilhelm S, Carter C, Lynch M, et al. Discovery and development of sorafenib: a multikinase inhibitor for treating cancer. Nat Rev Drug Discov. 2006;5:835-44.

45. Wang Y, Gao JC, Zhang D, et al. New insights into the antifibrotic effects of sorafenib on hepatic stellate cells and liver fibrosis. J Hepatol. 2010;53:132-44.

46. Su TH, Shiau CW, Jao P, et al. Sorafenib and its derivative SC-1 exhibit antifibrotic effects through signal transducer and activator of transcription 3 inhibition. Proc Natl Acad Sci USA. 2015;112: $7243-8$. 